\title{
GLOBALLY CONVERGENT SERIES FOR THE DIRICHLET ETA AND BETA FUNCTIONS
}

\author{
SUMIT KUMAR JHA
}

ABSTRACT. We derive the the following globally convergent series for the Dirichlet eta and beta functions

$$
\begin{aligned}
& \eta(s)=\sum_{k=0}^{\infty} \frac{2^{s}}{2^{\frac{k+1}{2}}} \cos \left(\frac{(k+1) \pi}{4}\right) \sum_{m=0}^{k}\left(\begin{array}{l}
k \\
m
\end{array}\right) \frac{(-1)^{m}}{(m+1)^{s}}, \\
& \beta(s)=\sum_{k=0}^{\infty} \frac{1}{2^{\frac{k+1}{2}}} \sin \left(\frac{(k+1) \pi}{4}\right) \sum_{m=0}^{k}\left(\begin{array}{l}
k \\
m
\end{array}\right) \frac{(-1)^{m}}{(m+1)^{s}} .
\end{aligned}
$$

\section{MAIN RESULTS}

Let $\eta(s)$ represent the Dirichlet eta function. H. Hasse ([1], [2]) proved that

$$
\eta(s)=\sum_{n=0}^{\infty} \frac{1}{2^{n+1}} \sum_{k=0}^{n}\left(\begin{array}{l}
n \\
k
\end{array}\right) \frac{(-1)^{k}}{(k+1)^{s}}
$$

We prove the following.

Theorem 1. Let $\eta(s)$ and $\beta(s)$ represent the Dirichlet eta function, and the Dirichlet beta function, respectively. Then we have

$$
\begin{aligned}
& \eta(s)=\sum_{k=0}^{\infty} \frac{2^{s}}{2^{\frac{k+1}{2}}} \cos \left(\frac{(k+1) \pi}{4}\right) \sum_{m=0}^{k}\left(\begin{array}{l}
k \\
m
\end{array}\right) \frac{(-1)^{m}}{(m+1)^{s}}, \\
& \beta(s)=\sum_{k=0}^{\infty} \frac{1}{2^{\frac{k+1}{2}}} \sin \left(\frac{(k+1) \pi}{4}\right) \sum_{m=0}^{k}\left(\begin{array}{l}
k \\
m
\end{array}\right) \frac{(-1)^{m}}{(m+1)^{s}} .
\end{aligned}
$$

Proof. The polylogarithm function, $\mathbf{L i}_{s}(z)$, has the following series representation [3, Theorem 2.1]

$$
\mathbf{L i}_{s}(z)=\sum_{k=0}^{\infty}\left(\frac{-z}{1-z}\right)^{k+1} \sum_{m=0}^{k}\left(\begin{array}{l}
k \\
m
\end{array}\right) \frac{(-1)^{m+1}}{(m+1)^{s}},
$$

for all $s, z \in \mathbb{C}$ with $\Re(z)<1 / 2$. Now, recalling that

$$
\mathbf{L i}_{s}(-i)=-2^{-s} \eta(s)-i \beta(s),
$$

we can derive equations (1) and (2) by taking real and imaginary parts in the series expression of $\mathbf{L i}_{s}(-i)$, respectively. 


\section{REFERENCES}

1. Hasse, Helmut (1930). Ein Summierungsverfahren fãijr die Riemannsche $\zeta$-Reihe [A summation method for the Riemann $\zeta$ series]. Mathematische Zeitschrift (in German). 32 (1): 458-464. doi:10.1007/BF01194645

2. Sondow, Jonathan (1994). Analytic continuation of Riemann's zeta function and values at negative integers via Euler's transformation of series. Proceedings of the American Mathematical Society. 120 (2): 421-424. doi:10.1090/S0002-9939-1994-11729547.

3. J. Guillera, J. Sondow, Double integrals and infinite products for some classical constants via analytic continuations of Lerch's transcendent, J. Ramanujan J (2008) 16: 247. https://doi.org/10.1007/s11139-007-9102-0

INTERNATIONAL INSTITUTE OF INFORMATION TECHNOLOGY, HYDERABAD, INDIA

E-mail address: kumarjha. sumit@research.iiit.ac. in 